\title{
The first instability in spherical Taylor-Couette flow
}

\author{
By GEZA SCHRAUF† \\ Applied Mathematics, California Institute of Technology, Pasadena, CA 91125, USA
}

(Received 24 June 1985 and in revised form 24 September 1985)

In this paper continuation methods are applied to the axisymmetric Navier-Stokes equations in order to investigate how the stability of spherical Couette flow depends on the gap size $\sigma$. We find that the flow loses its stability due to symmetry-breaking bifurcations and exhibits a transition with hysteresis into a flow with one pair of Taylor vortices if the gap size is sufficiently small, i.e. if $\sigma \leqslant \sigma_{B}$.

In wider gaps, i.e. for $\sigma_{\mathrm{B}}<\sigma \leqslant \sigma_{\mathrm{F}}$, both flows, the spherical Couette flow and the flow with one pair of Taylor vortices, are stable. We predict that the latter exists in much wider gaps than previous experiments and calculations showed. Taylor vortices do not exist if $\sigma>\sigma_{\mathrm{F}}$. The numbers $\sigma_{\mathrm{B}}$ and $\sigma_{\mathrm{F}}$ are computed by calculating the instability region of the spherical Couette flow and the region of existence of the flow with one pair of Taylor vortices.

\section{Introduction}

We consider a flow of a viscous incompressible fluid contained between two concentric spheres. The outer sphere is fixed while the inner one rotates. If the inner sphere rotates slowly the flow contains two large cells ranging from each pole to the equator. This flow is uniquely determined by the Reynolds number, and is called 'spherical Couette flow' (cf. figure 1). By increasing the Reynolds number, Khlebutin (1968), Sawatzki \& Zierep (1970), Wimmer (1976) and Belyaev et al. (1980) observed in their experiments a transition into a flow with one pair of Taylor vortices at the equator if the gap size $\sigma=\left(R_{2}-R_{1}\right) / R_{1}$ is less than 0.19. A transition back into spherical Couette flow occurs if the Reynolds number is reduced. No hysteresis was observed, i.e. both transitions occurred at the same Reynolds number.

The transition behaviour is found to depend strongly on the gap size $\sigma$. For $\sigma=0.24$, for example, Yavorskaya et al. (1980) observed a hysteresis in the transition to and from the flow with one pair of Taylor vortices. For $\sigma=0.30$ they were able to produce the Taylor-vortex flow by rotating both spheres and stopping the outer one when the vortices appeared. They observed a transition back into spherical Couette flow if the Reynolds number was decreased. However, the Taylor-vortex flow cannot be produced by rotating only the inner sphere. In that case, the spherical Couette flow remains stable until it encounters the so-called 'wide-gap instability' (Yavorskaya et al. 1980). No Taylor vortices have been observed for $\sigma \geqslant 0.40$.

Many more flow modes have been observed besides the spherical Couette flow and the flow with one pair of Taylor vortices. There are modes with more than one pair of Taylor vortices, with oscillating Taylor vortices, with wavy Taylor vortices, etc.

$\dagger$ Present address: MBB Transport- und Verkehrsflugzeuge, Abt. TE 213, Huenefeldstr. 1-5, 2800 Bremen 1, West Germany. 

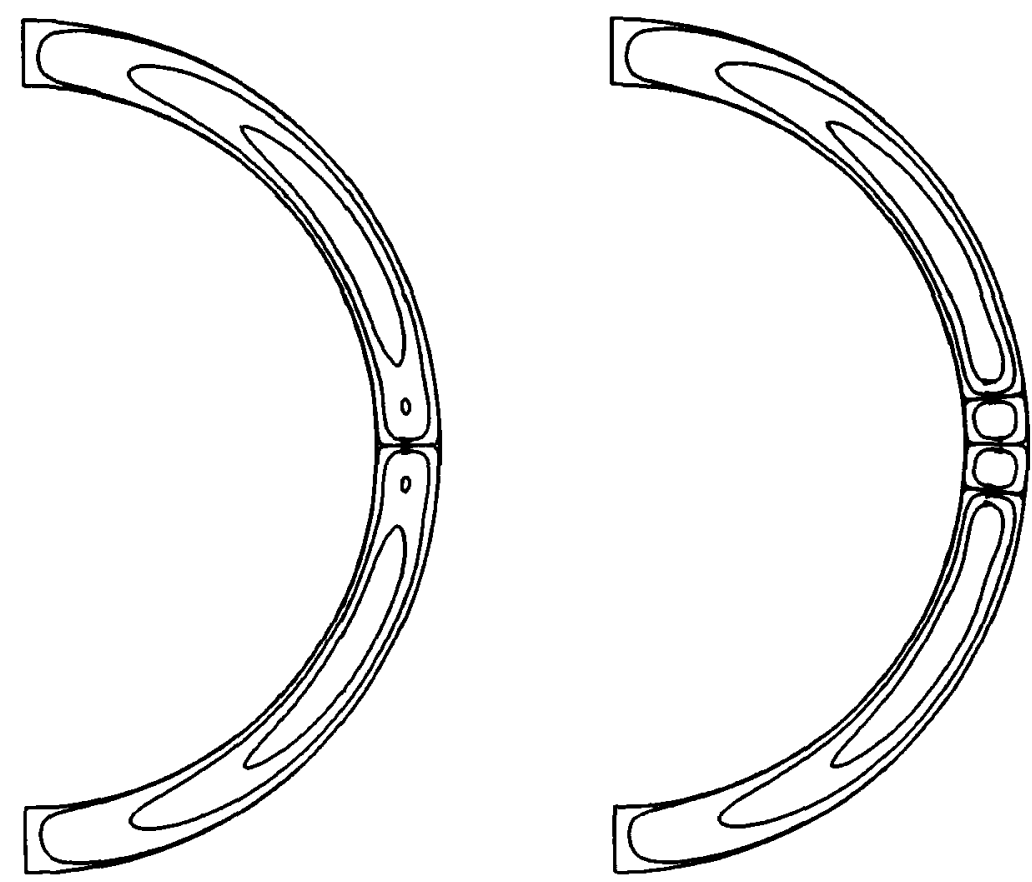

Figure 1. Streamlines of the spherical Couette flow at $R e=640$ and of the flow with one pair of Taylor vortices at $R e=660 . \sigma=0.18$.

The flow observed in the experiments is uniquely determined by the Reynolds number if the gap is sufficiently small or 'narrow'. However, it is non-unique, i.e. different flow modes can exist at the same Reynolds number, if the gap is 'medium-sized'. No Taylor vortices are detectable in 'wide' gaps.

We study the first transition, i.e. the transition from spherical Couette flow into the flow with one pair of Taylor vortices in medium-sized gaps, and investigate how the transition phenomena depend on the gap size $\sigma$.

In previous calculations (Schrauf 1982, 1983) the spherical Couette flow and the flow with one pair of Taylor vortices were obtained for several gap sizes by tracing the axially and equatorially symmetric solutions of the Navier-Stokes equations with a continuation method (Keller 1977). It is possible to identify the transition from Taylor-vortex into spherical Couette flow with a fold point of the solution branch. There was no indication for the reverse transition.

Tuckerman (1983) showed that the spherical Couette flow loses its stability owing to equatorially asymmetric disturbances. Without exploiting equatorial symmetry we detected a symmetry-breaking bifurcation point at which a branch with nonequatorially symmetric solutions with one pair of Taylor vortices bifurcates from the equatorially symmetric Couette flow. The Couette flow becomes unstable at the bifurcation point (Schrauf \& Krause 1984).

In this paper, the influence of the gap size on the first instability of spherical Couette flow has been investigated in order to explain the phenomena observed in the experiments and to determine the instability region of the spherical Couette flow and the region of existence of the flow with one pair of Taylor vortices. We hypothesize, furthermore, that the instability in the supercritical spherical Couette flow is caused by symmetry-breaking bifurcations. 


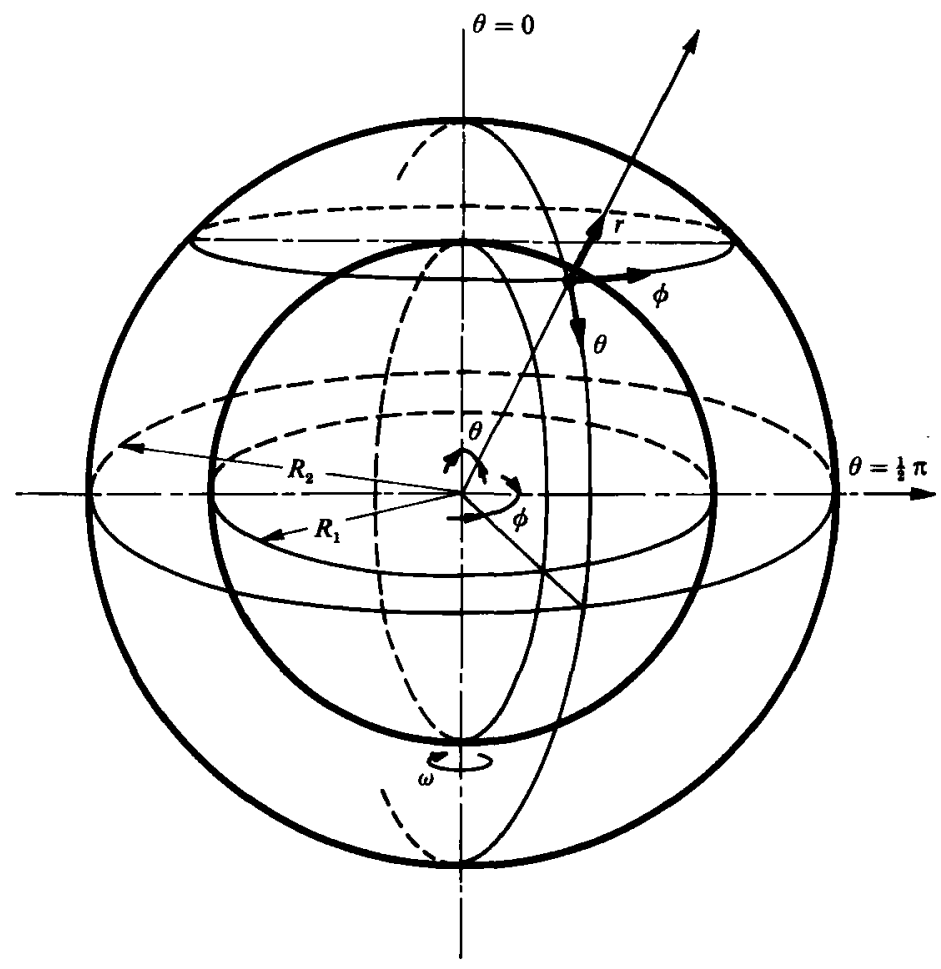

Fiaure 2. The geometry of the spherical annulus.

\section{Governing equations and numerical methods}

The geometry of the spherical annulus is shown in figure 2 . An incompressible fluid with constant kinematic viscosity $\nu$ fills the gap between the two concentric spheres with radii $R_{1}<R_{2}$. The inner sphere rotates with angular velocity $\omega$. Since we consider only axisymmetric flows, we can introduce a stream function $\tilde{\Psi}$, a vorticity function $\zeta$, and an angular velocity function $\Phi$ as follows (Krause \& Bartels 1980; Bonnet \& Alziary de Roquefort 1976; Munson \& Joseph 1971; Rosenhead 1963; Lamb 1932; Stokes 1842):

$$
\begin{gathered}
v^{r}(r, \theta)=\frac{1}{r \sin \theta} \frac{1}{r} \frac{\partial}{\partial \theta} \Psi(r, \theta), \\
v^{\theta}(r, \theta)=-\frac{1}{r \sin \theta} \frac{\partial}{\partial r} \Psi(r, \theta), \\
v^{\theta}(r, \theta)=\frac{1}{r \sin \theta} \Phi_{(r, \theta)} \\
(\nabla \times v)^{\phi}=\frac{1}{r}\left[\frac{\partial}{\partial r}\left(r v^{\theta}\right)-\frac{\partial}{\partial \theta} v^{r}\right]=\frac{1}{r \sin \theta} \xi(r, \theta) .
\end{gathered}
$$

Because the Taylor vortices occur near the equator, we rediscover the use of the variable $\tau=\cos \theta$ instead of the colatitude $\theta$. We define

$$
\Psi(r, \tau)=\Psi(r, \cos \theta)=\Psi(r, \theta),
$$


and analogously $\zeta(r, \tau)$ and $\Phi(r, \tau)$, and obtain the equations

$$
\begin{aligned}
&-\mathrm{D}^{2} \Phi+ \operatorname{Re}\left\{-\Psi_{\tau} \Phi_{r}+\Psi_{r} \Phi_{\tau}\right\}=0 \\
&-\mathrm{D}^{2} \zeta+\operatorname{Re}\left\{-\Psi_{\tau} \zeta_{r}+\Psi_{r} \zeta_{\tau}+\zeta\right. {\left.\left[\frac{2}{r} \Psi_{\tau}+\frac{2 \tau}{1-\tau^{2}} \Psi_{r}\right]-\Phi\left[\frac{2}{r} \Phi_{\tau}+\frac{2 \tau}{1-\tau^{2}} \Phi_{r}\right]\right\}=0 } \\
&-\mathrm{D}^{2} \Psi-r^{2} \zeta=0
\end{aligned}
$$

where

$$
\left.\begin{array}{c}
\mathrm{D}^{2}=r^{2} \frac{\partial^{2}}{\partial r^{2}}+\left(1-\tau^{2}\right) \frac{\partial^{2}}{\partial \tau^{2}}, \\
(\cdot)_{r}=\frac{\partial}{\partial r}, \quad(\cdot)_{\tau}=\frac{\partial}{\partial \tau} .
\end{array}\right\}
$$

Choosing $R_{1}$ as characteristic length and $\omega R_{1}$ as characteristic velocity, the Reynolds number is, thus,

$$
R e=\omega R_{1}^{2} / \nu .
$$

The advantages of using the variable $\tau$ instead of $\theta$ are that the operator $\mathrm{D}^{2}$ is symmetric and the matrix $\boldsymbol{F}_{u}$, which will appear later, has a much smaller dimension.

The domain on which we solve $(1 a-c)$ is

$$
\mathscr{D}(\alpha)=\left\{(r, \tau) \in \Re^{2}: \quad 1 \leqslant r \leqslant \alpha, \quad 1 \geqslant \tau \geqslant-1\right\}
$$

or, if only equatorially symmetric flows are considered,

$$
\mathscr{D}_{\mathbf{B}}(\alpha)=\left\{(r, \tau) \in \mathfrak{R}^{2}: \quad 1 \leqslant r \leqslant \alpha, \quad 1 \geqslant \tau \geqslant 0\right\} .
$$

$\alpha$ is defined as

$$
\alpha=\frac{R_{2}}{R_{1}}=\sigma+1 .
$$

Both computational domains have been incorporated into our code. The boundary conditions are Stokes' no-slip conditions at the spheres

$$
\begin{gathered}
\Phi(1, \tau)=1-\tau^{2}, \quad \Phi(\alpha, \tau)=0, \\
\Psi(1, \tau)=0, \quad \Psi(\alpha, \tau)=0, \\
\Psi_{r}(1, \tau)=0, \quad \Psi_{r}(\alpha, \tau)=0,
\end{gathered}
$$

and the symmetry conditions at the poles

$$
\begin{array}{cl}
\Phi(r, 1)=0, & \Phi(r,-1)=0, \\
\zeta(r, 1)=0, & \zeta(r,-1)=0, \\
\Psi(r, 1)=0, & \Psi(r,-1)=0 .
\end{array}
$$

The conditions with $\tau=-1$ are replaced by

$$
\Phi_{r}(r, 0)=0, \quad \zeta(r, 0)=0, \quad \Psi(r, 0)=0,
$$

if only equatorially symmetric flows are to be calculated.

There are no physical conditions for the vorticity function at the walls. Hence, we evaluate the Poisson equation (1c) and obtain

$$
\zeta(1, \tau)+\Psi_{r r}(1, \tau)=0, \quad \zeta(\alpha, \tau)+\Psi_{r r}(\alpha, \tau)=0 .
$$


The equations $(1 a-c)$ are discretized by central differences of second order. The conditions (5) and (7) are approximated either by Thom's formula

$$
\zeta_{1, j}-\frac{2}{\Delta r^{2}}\left\{\Psi_{1, j}-\Psi_{2, j}\right\}=0+O(\Delta r)
$$

or Jensen's formula

$$
\zeta_{1, j}-\frac{1}{2 \Delta r^{2}}\left\{7 \Psi_{1, j}-8 \Psi_{2, j}-\Psi_{3, j}\right\}=0+O\left(\Delta r^{2}\right)
$$

or Woods' formula

$$
\zeta_{1, j}+\frac{1}{2} \zeta_{2, j}-\frac{3}{\Delta r^{2}}\left\{\Psi_{1, j}-\Psi_{2, j}\right\}=0+O\left(\Delta r^{2}\right)
$$

where $\zeta_{i, j}=\zeta(1+(i-1) \Delta r,(j-1) \Delta \tau)$ and $\Psi_{i, j}$ respectively (Roache 1972). All three formulas are implemented in the code and can be interchanged easily. It can be shown (Schrauf 1983) that the system of equations $(1 b, c)$ and $(8)$ is equivalent to a second-order difference approximation of the fourth-order differential equation which is obtained by eliminating the vorticity function $\zeta$ from $(1 b)$ and $(1 c)$. Hence, second-order accuracy for the stream function is obtained if the equations $(1 b, c)$, and (8) are solved simultaneously, although formula (8) by itself is formally of first order. We found that only the discretization error, but not the bifurcation behaviour of the solutions, depends on the choice of the formula for the wall vorticity. Most of the calculations presented in this paper are done with Jensen's formula.

For computations on the smaller domain $\mathscr{D}_{\mathrm{s}}(\alpha)$, the Neumann condition $(6 a)$ for the angular velocity function $\Phi$ is approximated by

$$
\begin{aligned}
\frac{r_{i}^{2}}{\Delta r^{2}}\left\{-\Phi_{i-1, j}+2 \Phi_{i, j}-\Phi_{i+1, j}\right\} & +\frac{2}{\Delta \tau^{2}}\left\{-\Phi_{i, j-1}+\Phi_{i, j}\right\} \\
& -\frac{R e}{2 \Delta r \Delta \tau} \Psi_{i, j-1}\left\{\Phi_{i-1, j}-\Phi_{i+1, j}\right\}=0+O\left(\Delta r^{2}+\Delta \tau^{2}\right) .
\end{aligned}
$$

The difference equations form a system of nonlinear algebraic equations. They depend on the two parameters $R e$ and $\alpha$. For convenience we denote the system by

$$
\boldsymbol{F}(\boldsymbol{u}, R e, \alpha)=0,
$$

where $\boldsymbol{u}$ is the $N$-dimensional vector that consists of the values of the functions $\Phi, \zeta, \Psi$ at the gridpoints. The dimension of $u$ is

$$
N=(J-2)[3(I-2)+2]+I-2,
$$

where $I$ is the number of gridpoints in $r$-direction and $J$ the number in the $\tau$-direction.

The solution of the system (12) is a two-dimensional surface in $\mathfrak{R}^{N+2}$. Parts of this surface are calculated by determining its intersections with hyperplanes $R e=$ const. or $\alpha=$ const., i.e. by computing solution curves of

$$
F(u, R e, \alpha=\text { const. })=0, \quad F(u, R e=\text { const. }, \alpha)=0 .
$$

The folds of the surface (cf. figure 3 ) are calculated as well.

Because the problems $(13 a, b)$ both depend only on one parameter, they can be abbreviated

$$
\boldsymbol{F}(\boldsymbol{u}, \lambda)=0
$$

where $\lambda$ stands for either $R e$ or $\alpha$. 


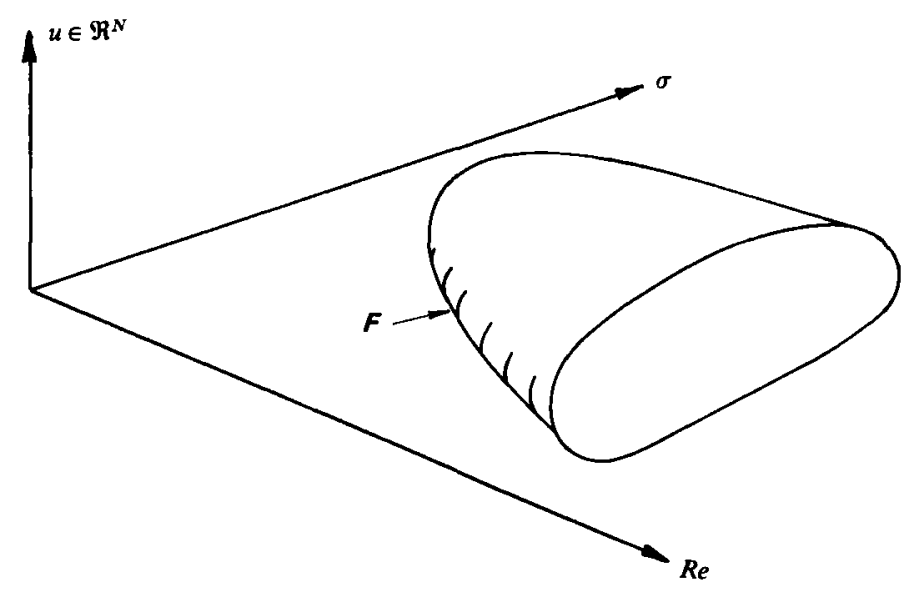

FIQURE 3. A two-dimensional surface in $\Re^{N+1+1}$ with a fold curve $F$.

The solution curves of (14) can be parameterized by $\lambda$. That is, we can try to calculate $\lambda \mapsto u(\lambda)$ with an Euler-Newton method. However, this method fails in fold points where the Jacobian $\boldsymbol{F}_{u}[u, \lambda]$ is singular. Therefore, a new parameter $s$ is introduced by

$$
\boldsymbol{N}[\boldsymbol{u}, \lambda, s]=\dot{u}\left(s_{0}\right) \cdot\left[\boldsymbol{u}(s)-\boldsymbol{u}\left(s_{0}\right)\right]+\dot{\lambda}\left(s_{0}\right)\left[\lambda(s)-\lambda\left(s_{0}\right)\right]-\left(s-s_{0}\right)=0
$$

(Keller 1977). The dot refers to differentiation with respect to s. Equation (15) is added to the system (14). The solution curves $s \mapsto(u(s), \lambda(s))$ of the larger system

$$
\boldsymbol{G}[x, s]=\left(\begin{array}{c}
\boldsymbol{F}(\boldsymbol{u}, \lambda) \\
\boldsymbol{N}(\boldsymbol{u}, \lambda, s)
\end{array}\right)=0, \quad x=(\boldsymbol{u}, \lambda),
$$

can be calculated by the Euler-Newton procedure

$$
\left.\begin{array}{c}
x^{(0)}(s)=x\left(s_{0}\right)+\left(s-s_{0}\right) \dot{x}\left(s_{0}\right), \\
\boldsymbol{G}_{x}\left[x^{(n)}(s), s\right] \delta x^{(n)}=-\boldsymbol{G}\left[x^{(n)}(s), s\right] \\
x^{(n+1)}(s)=\delta x^{(n)}+x^{(n)}(s)
\end{array}\right\}
$$

In each Newton step a linear system with the matrix

$$
\boldsymbol{G}_{x}=\left(\begin{array}{cc}
\boldsymbol{F}_{u} & \boldsymbol{F}_{\lambda} \\
\boldsymbol{N}_{u} & \boldsymbol{N}_{\lambda}
\end{array}\right)
$$

must be solved. This is equivalent to solving two systems with the matrix $\boldsymbol{F}_{u}$, so that the structure of $\boldsymbol{F}_{u}$ can be exploited. This works even at fold points, where $\boldsymbol{G}_{x}$ is regular but $\boldsymbol{F}_{u}$ is singular (Keller 1982).

In order to calculate a fold of the solution surface, i.e. a curve consisting of fold points, the system

$$
\boldsymbol{F}(u, \lambda, \tau)=0
$$

is augmented by adding the definition of a simple fold of a solution curve of $\boldsymbol{F}(u, \lambda, \tau=$ const. $)=0$ :

$$
\left(\boldsymbol{F}_{u}(u, \lambda, \tau)\right)^{\mathbf{T}} \psi=0, \quad\left(\boldsymbol{F}_{\lambda}(u, \lambda, \tau)\right)^{\mathbf{T}} \psi=1 .
$$

$(\lambda, \tau)$ is either $(R e, \alpha)$ or $(\alpha, R e)$ so that folds with respect to $R e$ or with respect to 
$\alpha$ can be calculated. Furthermore, an arclength parameter $s$ for the fold curve can be introduced by an additional equation

$$
\boldsymbol{N}(u, \lambda, \tau, s)=\mathbf{0}
$$

The equations $(20 a-d)$ form the nonlinear system

$$
\begin{gathered}
\boldsymbol{H}[y, s]=\left(\begin{array}{c}
\boldsymbol{F}(u, \lambda, \tau) \\
\boldsymbol{N}(u, \lambda, \tau, s) \\
\left(\boldsymbol{F}_{u}(u, \lambda, \tau,)\right)^{\mathbf{T}} \psi \\
\left(\boldsymbol{F}_{\lambda}(u, \lambda, \tau)\right)^{\mathbf{T}} \psi-1
\end{array}\right)=0 \\
y=(u, \lambda, \psi, \tau) \in \mathfrak{R}^{N+1+N+1}
\end{gathered}
$$

The fold curve $s \mapsto(u(s), \lambda(s), \tau(s))$ is a projection of the solution curve $s \mapsto y(s)=(u(s), \lambda(s), \psi(s), \tau(s))$ of $(21)$. The lattercan becalculated by an Euler-Newton method. A system with the matrix

$$
\boldsymbol{H}_{y}=\left(\begin{array}{cccc}
\boldsymbol{F}_{u} & \boldsymbol{F}_{\lambda} & 0 & \boldsymbol{F}_{\tau} \\
\boldsymbol{N}_{u} & \boldsymbol{N}_{\lambda} & 0 & \boldsymbol{N}_{\tau} \\
\left(\boldsymbol{F}_{u}^{\mathbf{T}} \psi\right)_{u} & \left(\boldsymbol{F}_{u}^{\mathbf{T}} \psi\right)_{\lambda} & \boldsymbol{F}_{u}^{\mathbf{T}} & \left(\boldsymbol{F}_{\boldsymbol{n}}^{\mathbf{T}} \psi\right)_{\tau} \\
\left(\boldsymbol{F}_{\lambda}^{\mathbf{T}} \psi\right)_{u} & \left(\boldsymbol{F}_{\lambda}^{\mathbf{T}} \psi\right)_{\lambda} & \boldsymbol{F}_{\lambda}^{\mathbf{T}} & \left(\boldsymbol{F}_{\lambda}^{\mathbf{T}} \psi\right)_{\tau}
\end{array}\right)
$$

must be solved at each Newton step. In Schrauf, Fier \& Keller (1985) we show that this is equivalent to solving two systems with the matrix $\boldsymbol{F}_{u}$ and three with the transposed matrix $\boldsymbol{F}_{\boldsymbol{u}}^{\mathbf{T}}$, and that all five systems can be solved by performing only one LU-decomposition of $\boldsymbol{F}_{\boldsymbol{u}}$.

\section{The instability region of the spherical Couette flow}

Spherical Couette flow exhibits a transition into an axially and equatorially symmetric flow with one pair of Taylor vortices at the equator if $\sigma=\left(R_{2}-R_{1}\right) / R_{1} \leqslant 0.19$. The reverse transition occurs at approximately the same Reynolds number. No hysteresis is observed. The critical Reynolds number can be calculated by empirical formulas proposed by Khlebutin (1968),

$$
R e_{*}=49.0 \sigma^{-\frac{3}{2}}
$$

or Belyaev et al. (1980),

$$
R e_{*}=41.3(1+\sigma) \sigma^{-\frac{3}{2}}
$$

The transition does exhibit hysteresis for the larger gap size $\sigma=0.2413$. Belyaev et al. produced the flow with one pair of Taylor vortices for $\sigma=0.3038$. To obtain this flow, they rotated both spheres simultaneously and stopped the outer one when the Taylor vortices appeared. They could not generate this flow by rotating only the inner sphere. Taylor vortices have not yet been observed for $\sigma \geqslant 0.4$.

Previous calculations for $\sigma=0.1765$, or $\eta=R_{1} / R_{2}=0.85$ (Schrauf \& Krause 1984), showed that the transition from spherical Couette flow to the mode with one pair of Taylor vortices can be explained by a symmetry-breaking bifurcation (Point $P$ in figure 4). The transition back to the basic flow coincides with a fold point (Point A in figure 4) at a slightly lower Reynolds number. The resulting hysteresis, however, is too small to be observed in experiments.

We now study how the location of the symmetry-breaking bifurcation point varies 


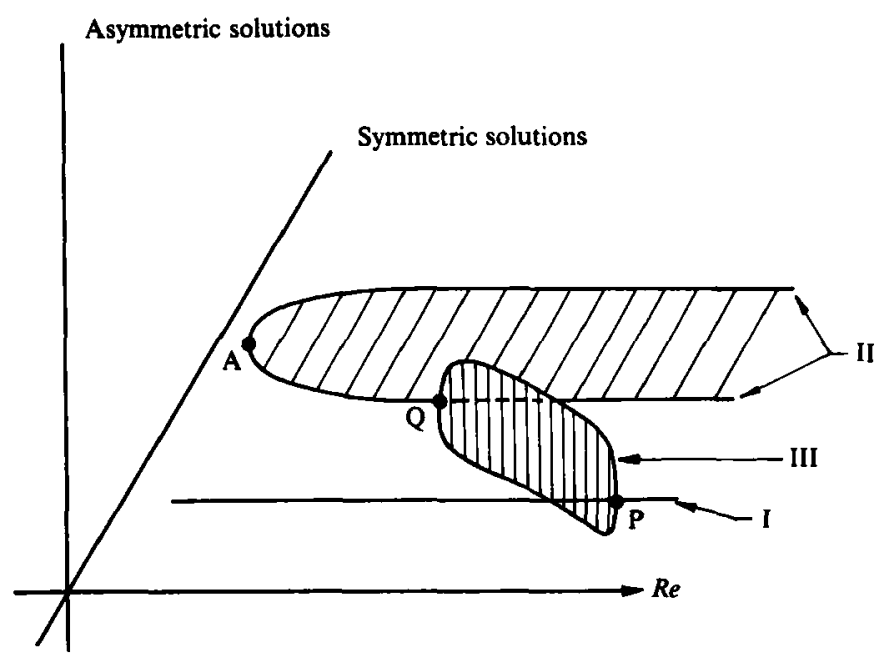

Figure 4. Bifurcation diagram taken from Schrauf \& Krause (1984). The solution branch I represents the spherical Couette flow, the branches II and III the equatorially symmetric and the non-equatorially symmetric flow with one pair of Taylor vortices.

with $\sigma$. From this, the region in the $(R e, \sigma)$-plane in which the spherical Couette flow is unstable can be determined. Then the curve with the fold points $A$ mentioned above is calculated in order to determine the boundary of the region in which the flow with one pair of Taylor vortices exists and is stable.

The finite-difference approximation perturbs the symmetry-breaking bifurcation of the spherical Couette flow. The calculated solution branches do not intersect as do the solid lines in figure 5, but behave like the dashed lines, i.e. the bifurcation is perturbed. For $\sigma=0.1800$ we calculate with $21 \times 121$ gridpoints

$$
R e_{1}=659.9720029 \quad R e_{2}=659.9720032
$$

and obtain

$$
|\Delta R e / R e| \approx 4.5 \cdot 10^{-10} \text {. }
$$

Although the perturbation of the bifurcation is hardly noticeable, it affects the choice of the numerical procedure because no available method allows following perturbed bifurcation points directly. Therefore, it is done indirectly by keeping one parameter constant and continuing in the other, i.e. we calculate solution branches of the problems (13a) or (13b). The bifurcation is detected by a change in the sign of the determinant of the Jacobian.

In the first series of calculations $\sigma$ is kept constant, and the solution branch of the spherical Couette flow is calculated using $21 \times 121$ gridpoints. This branch exhibits bifurcations for $\sigma \leqslant 0.2315$, but not for $\sigma=0.2320$. If the maximum gap size for which there is a bifurcation is denoted by $\sigma_{B}$, we find that $0.2315<\sigma_{B}<0.2320$. The loci of the bifurcation points are plotted as line $I$ in figure 6 . For $\sigma \leqslant 0.17$ we obtain good agreement with formula (24), shown as curve III. The Reynolds numbers of the computed bifurcation points and the ones calculated with formula (24) differ by less than $2.4 \%$. This difference increases to $5.2 \%$ for $\sigma=0.19$.

The upper part of curve $I$ is calculated as follows: Choosing $\sigma>\sigma_{B}$, we calculate the branch of the spherical Couette flow for this gap size. The points on this branch are then used as initial values for arclength continuation in $\alpha$, i.e. keeping the Reynolds number constant, we calculate the solution branches of $(13 b)$. These 


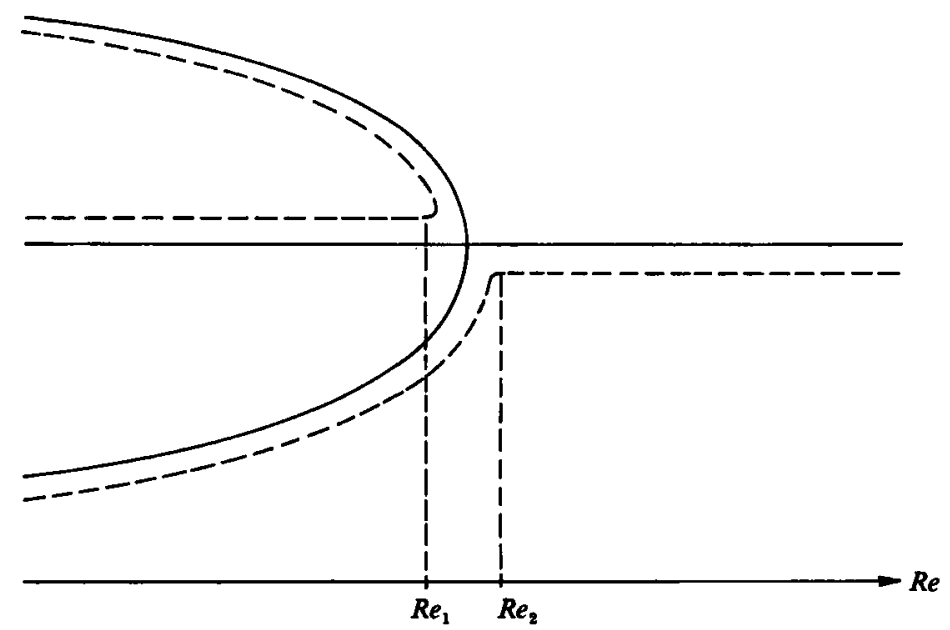

Figure 5. A perturbed bifurcation.

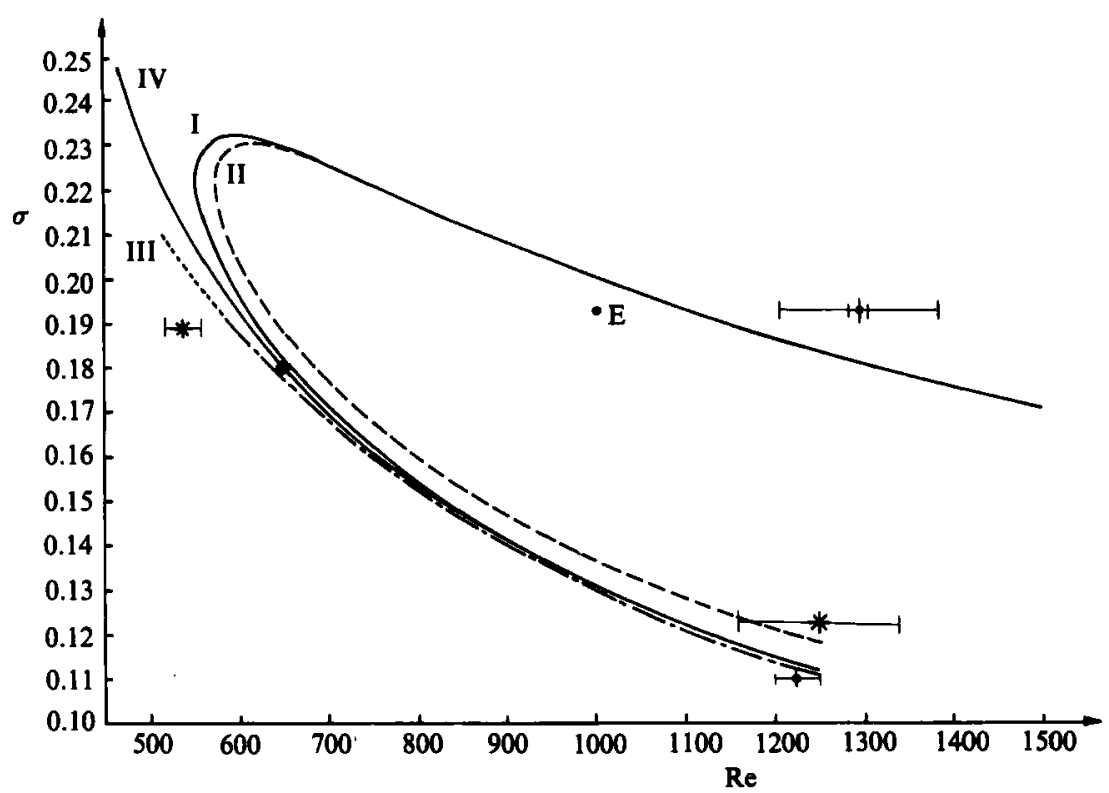

FraURe 6. Curves I and II are projections of symmetry-breaking bifurcation points calculated with $21 \times 121$ and $11 \times 61$ gridpoints respectively. Curve III shows the critical Reynolds numbers obtained with formula (24). Curve IV is the projection of a fold curve calculated with $21 \times 121$ gridpoints. *, Khlebutin (1968); $\bullet$, Wimmer (1976); †, Yavorskaya et al. (1980).

branches also have symmetry-breaking bifurcation points whose loci form the upper part of curve $I$.

In order to examine the influence of the mesh size, both series of calculations are repeated with $11 \times 61$ gridpoints. The results are plotted as curve II in figure 6 . The upper part of curve II coincides with curve I. The lower part of curve II is shifted towards higher Reynolds numbers, so that the region bounded by curve II is contained in the region bounded by curve $I$. It is expected that the latter region will be contained in the region determined by using more than $21 \times 121$ gridpoints. Thus, the corresponding region for the solution of the differential equations is a superset 


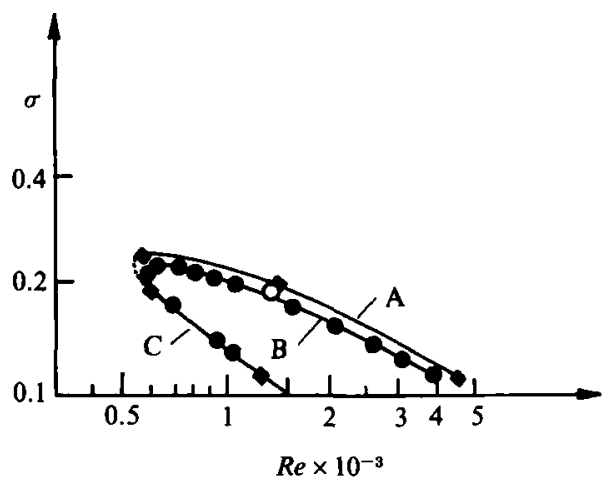

Fioure 7. The experimentally determined lower boundary of the region of existence of the supercritical basic flow, curve $A$, is compared with the projection of symmetry-breaking bifurcation points, curve $B$. Curve $C$ is the upper boundary of the region of existence of the subcritical Couette flow.

of the region bounded by curve I. As curve I consists of bifurcation points at which the spherical Couette flow becomes unstable to axisymmetric disturbances, we conclude that this flow is unstable, at least in the region bounded by curve $I$.

An axially and equatorially symmetric flow without Taylor vortices can be produced experimentally by a fast acceleration of the inner sphere. This flow is steady and is called 'supercritical spherical Couette flow' because it exists only at higher Reynolds numbers. It exhibits a transition into an oscillating mode if the Reynolds number is decreased quasi-stationarily.

In Schrauf \& Krause (1984), we calculated three branches of flows without Taylor vortices for $\sigma=0.1765$ and stated that one of them represents the supercritical Couette flow. We could only compare the calculated streamlines with those observed in experiments. By using arclength continuation in $R e$ and in $\sigma$ we see that the branch of the spherical Couette flow and the branch we chose belong to the same sheet of the solution surface in the $(u, R e, \sigma)$-space. This confirms that our previous choice was correct.

The supercritical Couette flow at $(R e, \sigma)=(1500.0,0.1765)$ can be connected with the spherical Couette flow at $(R e, \sigma)=(1.0,0.1765)$ by paths along which the flow remains stable with respect to axisymmetric disturbances. One path, for example, has a projection into the $(R e, \sigma)$-plane that consists of straight line segments connecting the vertices $(1.0,0.1765),(1.0,0.3000),(1500.0,0.3000),(1500.0,0.1765)$.

For each fixed $\sigma=\sigma_{0}<\sigma_{\mathrm{B}}$ the supercritical spherical Couette flow becomes unstable with respect to axisymmetric disturbances at the symmetry-breaking bifurcation point whose projection in the $(R e, \sigma)$-plane is given by the intersection of the line $\sigma=\sigma_{0}$ and the upper part of curve I. The question arises of whether this bifurcation describes the transition from this flow into the oscillating mode.

The most precise measurement of the transition Reynolds number was performed by I. M. Yavorskaya (1984, private communication). She determined $R e_{\mathrm{T}}=1294 \pm$ 10 for $\sigma=0.1924$. We calculated $R e_{\mathrm{B}}=1111.8$, which is much smaller than $R e_{\mathrm{T}}$. However the influence of imperfections in the spheres is very large since $\Delta R e / \Delta \sigma \approx$ 18000. Using her data, we estimate the relative error in $\sigma$ to be $0.5 \%$ and conclude that the error in the measured transition Reynolds number caused by the inexactness of the spheres is about $\Delta R e \approx 90$.

In figure 7 our results are compared with the few experimental results available. 
Curve $A$ is the lower boundary of the region in which the supercritical Couette flow is stable as determined by Belyaev et al. (1980). The three diamonds on the curve A indicate their measurements.

The open circle represents the new measurement of Yavorskaya. The loci of the symmetry-breaking bifurcations are shown as curve B. They follow qualitatively the experimentally determined curve. It can be assumed that the difference is caused by a systematic error. We hypothesize that the transition of the supercritical basic flow is described by the symmetry-breaking bifurcations. However, due to threedimensional effects, the instability region of the supercritical spherical Couette flow could be a superset of the region bounded by curve $I$.

In previous calculations a fold in the branch of the supercritical basic flow for $\sigma=0.1765$ was obtained (Point $E$ in figure 1 of Schrauf \& Krause 1984). In order to show that the transition cannot be caused by this fold point, we have calculated the corresponding fold for $\sigma=0.1924$ using equatorial symmetry and different grids:

$\begin{array}{cc}\text { gridpoints } & R e_{\mathbf{F}} \\ 11 \times 61 & 1065.3 \\ 16 \times 91 & 1029.2 \\ 21 \times 121 & 1017.6 \\ 31 \times 181 & 1009.7\end{array}$

We obtain $R e_{\mathrm{F}}=1003.4$ by Richardson extrapolation and insert $(R e, \sigma)=$ (1003.4,0.1924) as point $E$ in figure 6 to show that the Reynolds number of the fold point is less than the Reynolds number of the symmetry-breaking bifurcation point at which the stability changes. Therefore, the transition cannot be caused by the fold.

\section{The region of existence of the flow with one pair of Taylor vortices}

The solutions with one pair of Taylor vortices at the equator form a two-dimensional surface in $\Re^{N+1+1}$. This surface is bounded by a fold curve. The boundary of the region in which the flow with one pair of Taylor vortices exists is therefore the projection of the fold onto the $(R e, \sigma)$-plane.

The fold has been calculated with the algorithm described in $\$ 2$. Because we already know that the flow in the fold is symmetric with respect to the equator, the size of the matrix $\boldsymbol{F}_{u}$ can be reduced by restricting ourselves to equatorially symmetric flows. However, it is necessary to check all calculations by repeating them without exploiting equatorial symmetry.

Using $21 \times 121$ gridpoints, we calculate a fold whose projection onto the $(R e, \sigma)$-plane is plotted as curve IV in figure 8. The streamlines of several flows on the fold are plotted in figure 9. Without imposing equatorial symmetry, a slightly shifted curve, part of which is shown as curve IV in figure 6 , is obtained. If only $11 \times 61$ gridpoints and the symmetry condition are used, we obtain curve $V$ in figure 8 , which agrees well with curve IV for $\sigma \leqslant 0.2$. The difference in Reynolds number here is less than $0.3 \%$ but increases with $\sigma$. This is due to the fact that the radial mesh size $\Delta r=\Delta r(\sigma)$ increases with $\sigma$ if the number of radial gridpoints is kept constant, so that the difference approximation becomes worse. The solution of the difference equations fails to approximate the solution of the differential equations if $\sigma>0.5$ and only eleven radial gridpoints are used.

In order to analyse the behaviour of the fold if the mesh is refined, we calculate two fold points on the upper part of the fold curve with $41 \times 241$ gridpoints. The points 


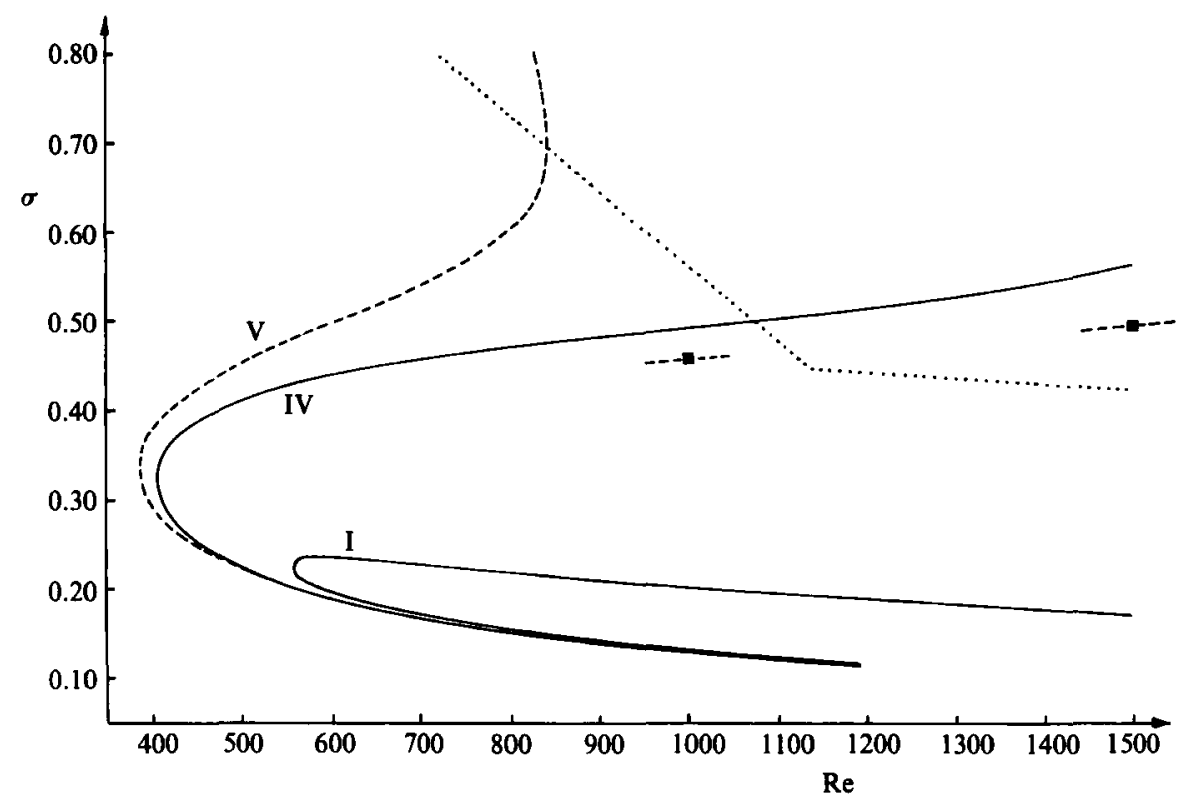

Figure 8. Curve $I$ is the projection of symmetry-breaking bifurcation points also shown in figure 6 . The curves IV and $V$ are projections of fold curves calculated with $21 \times 121$ and $11 \times 61$ gridpoints respectively. The two squares indicate fold points calculated with $41 \times 421$ gridpoints. The dotted line bounds the region of the wide-gap instability.

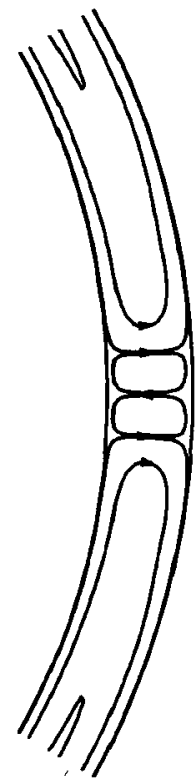

(a)

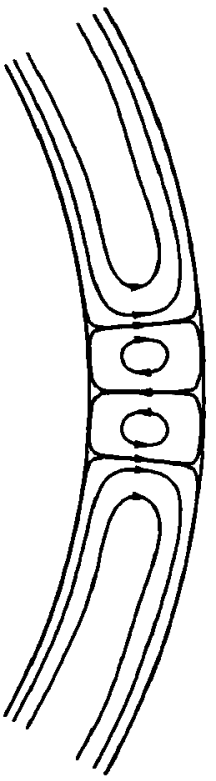

(b)

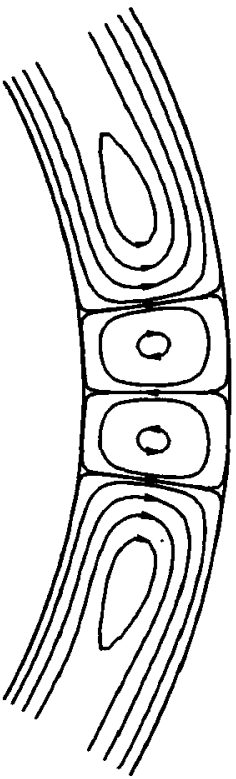

(c)

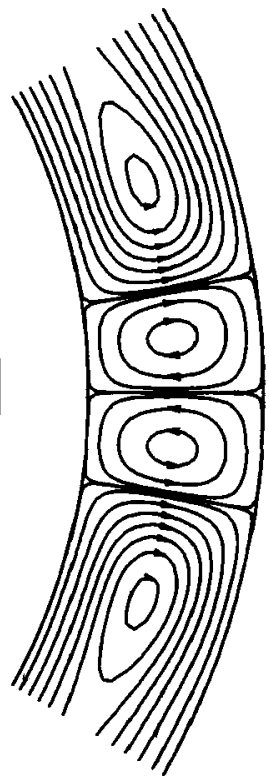

(d)

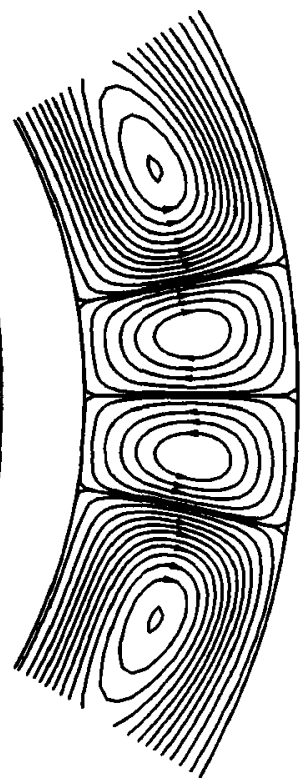

(e)

Figure 9. Streamlines of flows on the fold IV of figure 8 calculated with $21 \times 121$ gridpoints. '(a) $R e=1000, \sigma=0.1289 ;(b) 645,0.18 ;(c) 472,0.2413 ;(d) 409,0.3038 ;(e) 477,0.4$. 


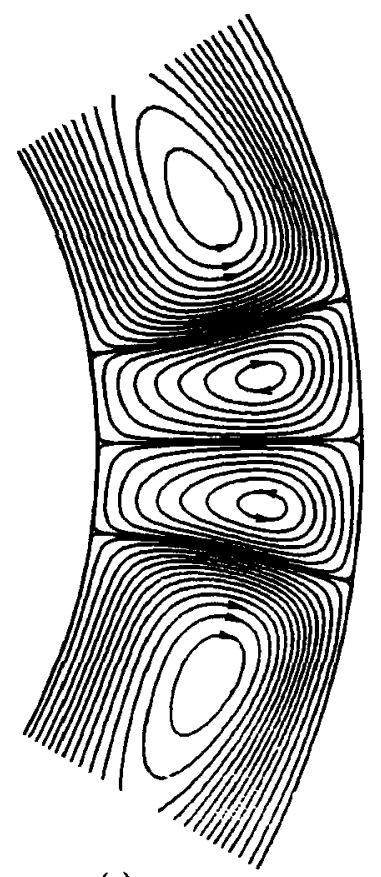

(a)

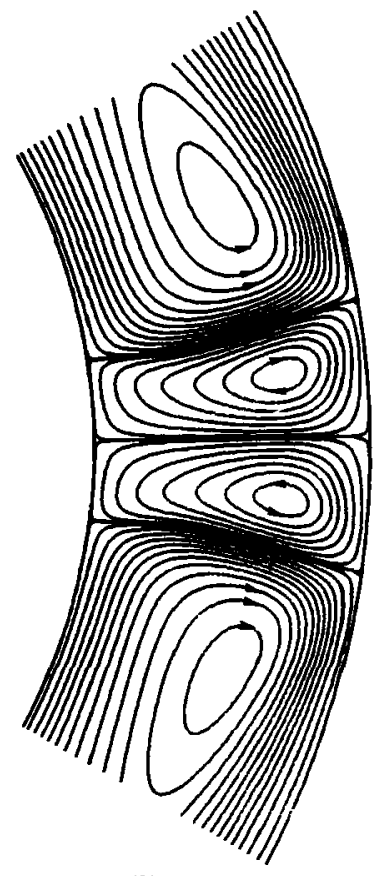

(b)

Fiaure 10. Streamlines of the two flows of figure 8 that are calculated with $41 \times 241$ gridpoints. (a) $R e=1000, \sigma=0.4673 ;(b) 1500,0.4963$.

are represented by squares in figure 8 ; the streamlines of the solutions are plotted in figure 10.

In order to study the topology of the solution surface, intersections with hyperplanes $R e=$ const. are determined by calculating solution curves of $(13 b)$. We find that these cuts are closed curves in $\Re^{N+1+1}$. The streamlines of the solutions along one of these loops are shown in figures 11-13.

If the solution surface is cut along the fold curve, we get two sides, side $A$ and side B. Side A consists of stable solutions which are observed in experiments if $\sigma \leqslant 0.3038$. For example, the first, second and third streamline plots in figure 12 are flows obtained also by Wimmer (1976) and Belyaev et al. (1980). However, the fourth plot shows a flow which, although not observed in experiments, is connected to the flow of the third plot by a solution curve which preserves stability. Therefore, the fourth flow either can be produced in experiments also, or is unstable due to three-dimensional disturbances. Side B consists of unstable solutions. The change of stability occurs at the fold points. The streamlines of the solutions in these points are plotted in figure 11 .

Yavorskaya et al. (1980) detected a weak, three-dimensional instability in spherical Couette flow. They called it ' wide-gap instability', because it occurs only in wide gaps, i.e. in gaps in which no Taylor vortex flow exists; in medium-sized gaps the spherical Couette flow becomes unstable at much lower Reynolds numbers, and there is a transition into the flow with one pair of Taylor vortices, as discussed above. The region in which the wide-gap instability appears is bounded by the dotted curve in figure 8.

Assuming that all solutions on side $A$ are stable, we estimate how far the fold curve must be calculated in order to determine the widest gap in which Taylor 


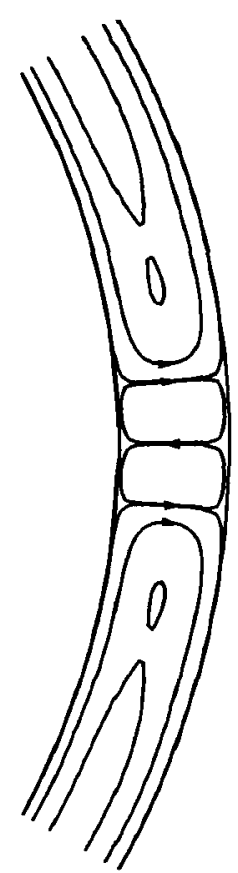

(a)

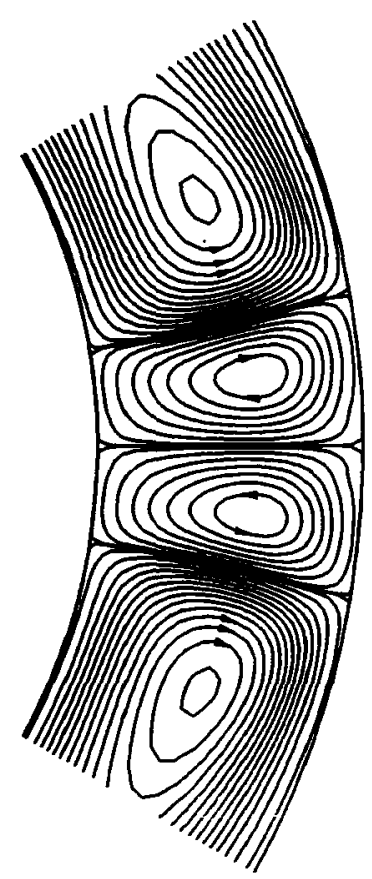

(b)

Frgure 11. Streamlines of the flows at the intersections of the loop at $R e=800$ with the fold curve. $(a) \sigma=0.152 ;(b) 0.4695$.

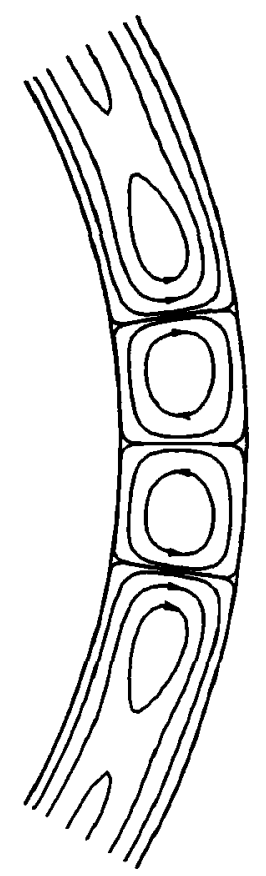

(a)

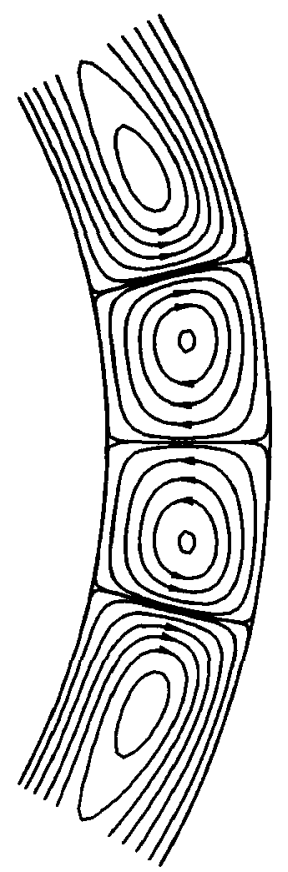

(b)

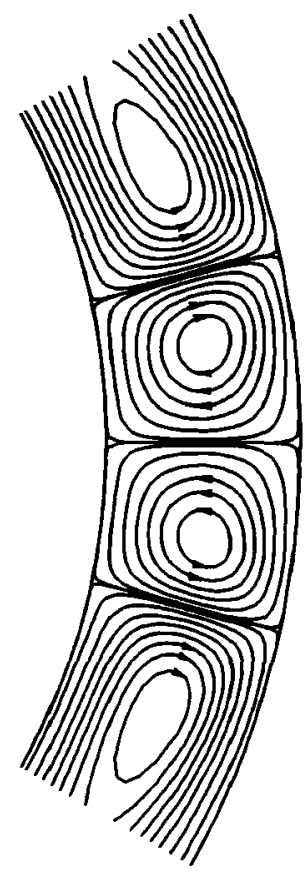

(c)

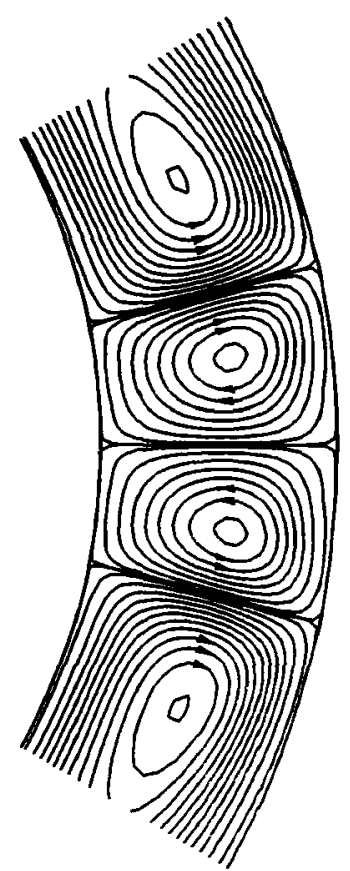

(d)

Figure 12. Streamlines of flows on the stable side of the loop at $R e=800 .(a) \sigma=0.18$; (b) $0.2413 ;(c) 0.3038 ;(d) 0.4$. 


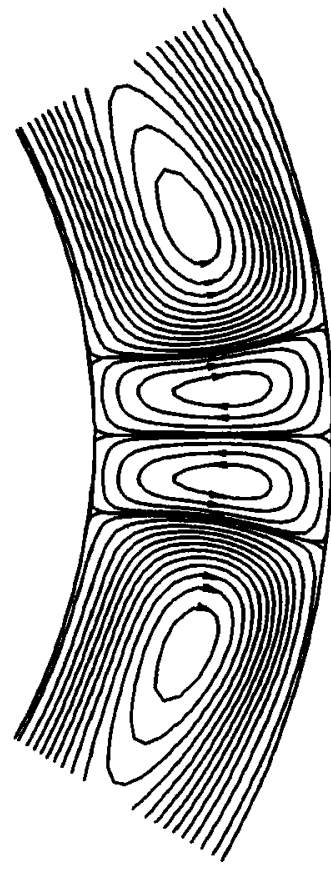

(a)

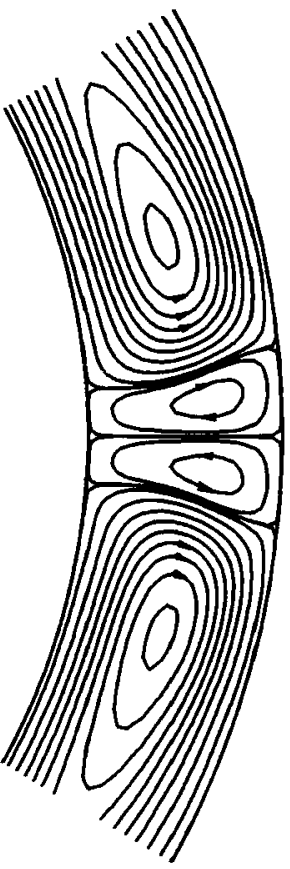

(b)

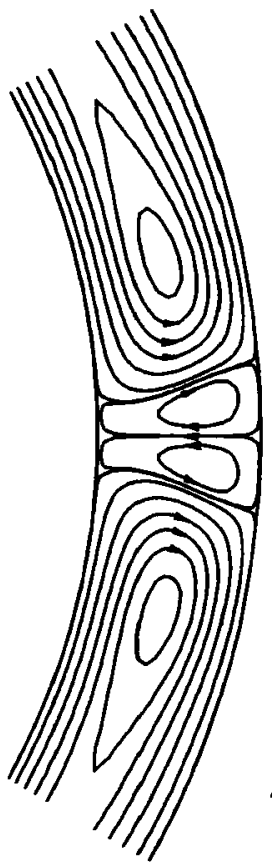

(c)

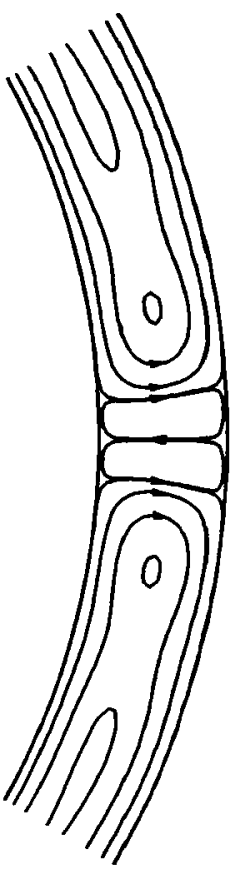

(d)

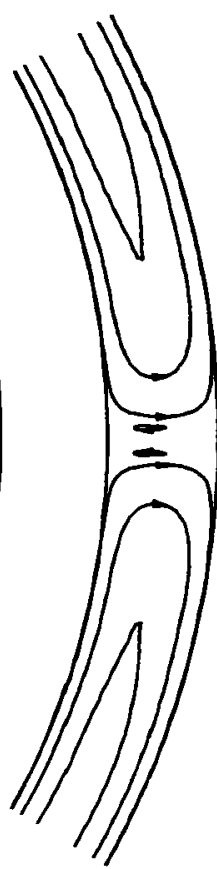

(e)

Figure 13. Streamlines of flows on the unstable side of the loop at $R e=800$. (a) $\sigma=0.4$; (b) $0.3038 ;(c) 0.2413 ;(d) 0.18 ;(e) 0.1539$.

vortices appear. A reasonable assumption is that the flow with one pair of Taylor vortices becomes unstable due to three-dimensional disturbances at Reynolds numbers near the critical Reynolds numbers of the wide-gap instability. Therefore it is sufficient to calculate the upper part of the fold curve IV in figure 8 up to $R e=1500$. We use the two fold points with $41 \times 241$ gridpoints, improve their $\sigma$-coordinates by Richardson extrapolation, and obtain $(R e, \sigma)=(1000.0,0.4587)$ and $(1500.0,0.4742)$. Hence, the widest gap for which the flow with one pair of Taylor vortices exists has a width of $\sigma_{F} \approx 0.45-0.48$.

The region of existence of this flow is larger than the instability region of the spherical Couette flow, and we obtain the following results:

In gaps with $\sigma \leqslant \sigma_{\mathrm{B}}, 0.2315<\sigma_{\mathrm{B}} \leqslant 0.24$, the spherical Couette flow becomes unstable and exhibits a transition into the flow with one pair of Taylor vortices. The transition back to the spherical Couette flow occurs at a lower Reynolds number, so that there is hysteresis.

For $\sigma_{\mathrm{B}}<\sigma \leqslant \sigma_{\mathrm{F}}$ the spherical Couette flow remains stable (until it encounters the wide-gap instability). The flow with one pair of Taylor vortices also exists; however, it cannot be produced by rotating only the inner sphere.

Taylor vortices do not exist if $\sigma>\sigma_{\mathrm{F}}$.

We calculate $\Delta R e$ for the hysteresis mentioned above by comparing the Reynolds number $R e_{\mathrm{B}}$ of the symmetry-breaking bifurcation points with the Reynolds number $R e_{\mathrm{F}}$ of the fold for the same gap size $\sigma \leqslant \sigma_{\mathrm{B}}$. The results are shown in table 1.

The only hysteresis that was observed in the experiments is the one reported by Belyaev et al. (1980) for the gap size $\sigma=0.2413$. They determined the Reynolds number of the transition to the flow with one pair of Taylor vortices as $R e=520$ and 


\begin{tabular}{crrr}
$\sigma$ & \multicolumn{1}{c}{$R e_{\mathrm{F}}$} & \multicolumn{1}{c}{$R e_{\mathrm{B}}$} & $\Delta R e$ \\
0.1150 & 1200.0 & 1201.3 & 1.3 \\
0.1222 & 1100.0 & 1101.5 & 1.5 \\
0.1306 & 1000.0 & 1001.8 & 1.8 \\
0.1409 & 900.0 & 902.2 & 2.2 \\
0.1536 & 800.0 & 803.1 & 3.1 \\
0.1702 & 700.0 & 705.2 & 5.2 \\
0.1931 & 600.0 & 611.5 & 11.5 \\
0.2030 & 566.7 & 583.4 & 16.7 \\
0.2147 & 533.3 & 560.5 & 27.2 \\
0.2289 & 500.0 & 561.9 & 61.9
\end{tabular}

TABLE 1. The Reynolds numbers of the fold and of the symmetry-breaking bifurcation point calculated with $21 \times 121$ grid points on the full gap.

the Reynolds number for the transition back to spherical Couette flow as $R e=463$. For this hysteresis we have $\Delta R e=57$.

With $21 \times 121$ gridpoints we obtain for $\sigma=0.2290$ a hysteresis with $\Delta R e=62$. Here we reach qualitative agreement. However, it is important to recall that the curves I and IV in figure 6 shift if more gridpoints are used.

Recently, Yavorskaya (1984, private communication) repeated the experiment and detected a hysteresis with $\Delta R e=0.70 \pm 0.25$ for the gap size $\sigma=0.1096$. Again, we have qualitative agreement with our results. However, the parameters of her experiment lie in the region $\sigma \leqslant 0.15$ and $R e \geqslant 1200$. This region has been excluded from our presentation because the solution surface with one pair of Taylor vortices interacts in a very complicated manner with two more sheets.

I thank John Bolstadt and Herb Keller for many fruitful discussions. This work was supported by the Deutsche Forschungsgemeinschaft (research scholarship no. Schr271/1-2) and by the U.S. Department of Energy (contract no. DE-AM0376 SF00761.

\section{REFERENCES}

Belyaev, Yu. N., Monakhov, A. A., Khlebutin, G. N. \& Yavorskaya, I. M. 1980 Issledovanie ustoichivosti i needinstvennosti techenii vo vraschayuschikhsya sfericheskikh sloyakh (Investigation of stability and nonuniqueness of the flow between rotating spheres). Rep. No. 567, Space Research Institute of the Academy of Science, USSR.

Bartels, F. 1982 Taylor vortices between two concentric rotating spheres. J. Fluid Mech. 19, 1.

Bonnet, J.-P. \& Alziary de Roquefort, T. 1976 Écoulement entre deux sphères concentriques en rotation. J. Mec. 13, 373.

KeLLER, H. B. 1977 Numerical solution of bifurcation and nonlinear eigenvalue problems. In Applications of Bifurcation Theory (ed. P. Rabinowitz). Academic.

KeLler, H. B. 1982 Practical procedures in path following near limit points. In Proc. 5th Intl Symp. on Computing Methods in Applied Sciences and Engineering, Versailles (eds. R. Glowinsky \& J. L. Lions), p. 177. North-Holland.

KhLEBUTIN, G. N. 1968 Stability of fluid motion between a rotating and a concentric sphere. Fluid Dyn. 3, 31.

KraUSe, E. \& Bartels, F. 1980 Finite-difference solutions of the Navier-Stokes equations for axially symmetric flows in spherical gaps. In Approximation Methods for Navier-Stokes Problems, Proc. IUTAM Symp., Paderborn (eds. E. Dold \& B. Eckermann), p. 313.

Lams, H. 1932 Hydrodynamics. Cambridge University Press. 
Munson, B. R. \& Joseph, D. D. 1971 Viscous incompressible flow between concentric rotating spheres. Part 1. Basic flow. J. Fluid Mech. 49, 289.

ROACHe, P. J. 1972 Computational Fluid Dynamics. Hermosa.

Rosenhead, L. 1963 Laminar Boundary Layers. Oxford University Press.

SawatZki, O.\&Zierep, J. 1970 DasStromfeld im Spaltzwischen zwei konzentrischen Kugelfächen, von denen die innere rotiert. Acta Mechanica 9, 13.

Schraur, G. 1982 Branching of Navier-Stokes equation in a spherical gap. In 8th Intl Conf. on Numerical Methods in Fluid Dynamics (ed. E. Krause). Lecture Notes in Physics, vol. 170, p. 474. Springer.

SchraUF, G. 1983 Lösungen der Navier-Stokes Gleichungen für stationäre Strömungen im Kugelspalt. Ph.D. thesis, University of Bonn.

Schrauf, G., Fier, J. M. \& Krller, H. B. 1985 Fold continuation in the Taylor Problem (in preparation).

SChraUf, G. \& KRAUSE, E. 1984 Symmetric and asymmetric Taylor vortices in a spherical gap. In The 2nd IUTAM Symp. on Laminar-Turbulent Transition (ed. V. V. Kozlov), p. 659. Springer.

Stokes, G. G. 1842 On the steady motion of incompressible fluids. Trans. Camb. Phil. Soc. 7, 439.

Tuckerman, L. S. 1983 Formation of Taylor vortices in spherical Couette flow. Ph.D. thesis, Massachusetts Institute of Technology.

Wimmer, M. 1976 Experiments on a viscous fluid flow between concentric rotating spheres. J. Fluid Mech. 78, 317.

Yavorskaya, I. M., Belyaev, Yu. N., Monakhov, A. A., Astaf'eva, N. M., Scherbakov, S. A. \& VvedenskaYA, N. D. 1980 Stability, non-uniqueness and the transition to turbulence in the flow between two rotating spheres. Rep. No. 595, Space Research Institute of the Academy of Science, USSR. 